\title{
Dendritic Cell Immunotherapy, the Next Step in Cancer Treatment
}

\author{
Teunis B H Geijtenbeek ${ }^{1, *}$ \\ ${ }^{1}$ Department of Experimental Immunology, Academic Medical Center, University of \\ Amsterdam, Amsterdam, The Netherlands \\ * Corresponding author: Teunis B H Geijtenbeek, Department of Experimental Immu- \\ nology, Academic Medical Center, University of Amsterdam, Meibergdreef 9, 1105 AZ, \\ Amsterdam, The Netherlands.E-mail: t.b.geijtenbeek@amc.uva.nl
}

DOI: $10.21859 /$ mci-01025

Submitted: 24 September 2016

Revised: 21 October 2016

Accepted: 3 December 2016

ePublished: 8 March 2017

Keywords:

Dendritic Cells

Immunotherapy

Neoplasms

(C) 2017. Multidisciplinary Cancer Investigation

Cancer immunotherapy has gained a lot of interest over the last few years due to the success of immune checkpoint inhibitors in treating cancer $[1,2]$. Immune checkpoint inhibitors, such as monoclonal antibodies against cytotoxic T-lymphocyte-associated antigen 4 (CTLA-4) and programmed death 1 (PD-1) have been shown to increase survival of patients with advanced cancers $[1,2]$. These inhibitors rely on preventing immunosuppression and enhancing anti-tumor responses. Under healthy conditions, over-activation of T cell immunity is controlled by immune checkpoints, which are molecules that suppress $\mathrm{T}$ cell function and thereby prevent uncontrolled $\mathrm{T}$ cell activation and autoimmunity. However, tumor cells overexpress or induce these checkpoint molecules leading to inhibition of anti-tumor $\mathrm{T}$ cell function, which contributes to the development of cancer tolerance in $T$ cells cancers $[1,2]$. The immune checkpoint inhibitors prevent cancer-induced immune suppression and thereby restore the function of $\mathrm{T}$ cells that are programmed and trained to fight against tumor cells. However, abrogating immune suppression is not always sufficient, as anti-tumor $\mathrm{T}$ cell immunity might be severely hampered or even absent in patients with cancer. Therefore, there is an urgent need for novel cancer therapies that induce anti-tumor responses in patients to enhance the effect of immune checkpoint inhibitors.

Dendritic cells (DCs), as professional antigen-presenting cells, are vital for the activation of immune responses and therefore DC-based cancer immunotherapy can be a very powerful method to induce anti-tumor immune responses in patients [3]. This immunotherapy is based on training autologous DCs from patients to induce anti-tumor immunity. Several studies have shown that DC-based immunotherapy is safe and can improve survival of patients with cancer by eliciting anti-tumor immunity [3]. Although proof-of-principle has been provided for DC-based cancer immunotherapy, the clinical success has been suboptimal [3]. Recent advances in our understanding of DC immunobiology have provided us with better methods to train DCs to activate powerful anti-tumor immunity, which depends on robust activation of $\mathrm{T}$ helper cells to propagate anti-tumor immunity as well as cytotoxic T cells for killing tumor cells [4]. DC-based immunotherapy consists of different steps; the ex vivo generation of DCs, tumor loading of DCs and DC activation. The ex vivo generation of monocyte-derived DCs from autologous monocytes from patients is well developed and commonly used [3]. It is worthy of note that DCs consist of different subsets with distinct functions and certain DC subsets are more efficient in inducing anti-tumor immunity than others. Recent advances allow the generation of different DC subsets from monocytes, which might be very powerful in DC-based immunotherapy [5]. Next, the ex vivo generated DCs need to be trained to present tumor antigens to $\mathrm{T}$ cells, the so-called tumor antigen loading. DCs can be loaded via different methods such as with recombinant tumor antigens or transfected with RNA from tumor cells, the former can be standardized but might allow tumor escape due to its high specificity, whereas the use of tumor cell RNA will allow for presentation of several tumor antigens and broadens the specificity considerably [4]. The next step is the activation of DCs, which is required to program the DCs for efficient activation of anti-tumor $\mathrm{T}$ helper cells and cytotoxic T cells, the crucial effector cells in anti-tumor immunity. This is the decisive step in the induction of anti-tumor immunity, since DCs can be activated by different stimuli, leading to very distinct immune responses. Our understanding of DC activation and induction of immune responses has increased in the last decade and allows us to specifically activate 
DCs to induce the correct $\mathrm{T}$ helper cell and cytotoxic $\mathrm{T}$ cell response to fight cancer. In particular, it has become clear that DC activation program can be tailored to the pathogen or tumor by triggering a specific combination of receptors, such as Toll-like receptors, C-type lectin receptors, and cytokine receptors [6]. Our knowledge about these receptors and the functional consequences of different DC maturation steps provides us with powerful tools to develop novel DC activation methods that lead to effective anti-tumor immunity.

Therefore, as immune checkpoint inhibitors prevent immune suppression by the tumor, DC-based immunotherapy can be the next important step in the development of effective cancer immunotherapy to induce powerful anti-tumor immunity. The specific programming of DCs for anti-tumor activity is an exciting new field that should be further developed to improve the success of DC-based immunotherapy in treating patients with cancer.

\section{REFERENCES}

1. La-Beck NM, Jean GW, Huynh C, Alzghari SK, Lowe DB. Immune Checkpoint Inhibitors: New Insights and Current Place in Cancer Therapy. Pharmacotherapy. 2015;35(10):963-76. DOI: 10.1002/phar.1643 PMID: 26497482

2. Buchbinder E, Hodi FS. Cytotoxic T lymphocyte antigen-4 and immune checkpoint blockade. J Clin Invest. 2015;125(9):337783. DOI: $10.1172 / J C I 80012$ PMID: 26325034

3. Anguille S, Smits EL, Lion E, van Tendeloo VF, Berneman ZN. Clinical use of dendritic cells for cancer therapy. Lancet Oncol. 2014;15(7):e257-67. DOI: 10.1016/S1470-2045(13)70585-0 PMID: 24872109

4. Chiang CL, Hagemann AR, Leskowitz R, Mick R, Garrabrant T, Czerniecki BJ, et al. Day-4 myeloid dendritic cells pulsed with whole tumor lysate are highly immunogenic and elicit potent anti-tumor responses. PLoS One. 2011;6(12):e28732. DOI: 10.1371/journal.pone.0028732 PMID: 22194898

5. Sachamitr P, Hackett S, Fairchild PJ. Induced pluripotent stem cells: challenges and opportunities for cancer immunotherapy. Front Immunol. 2014;5:176. DOI: 10.3389/fimmu.2014.00176 PMID: 24860566

6. Geijtenbeek TB, Gringhuis SI. Signalling through C-type lectin receptors: shaping immune responses. Nat Rev Immunol. 2009;9 (7):465-79. DOI: 10.1038/nri2569 PMID: $19521399 t$ 